\title{
Rapid Coating of Aqueous Pearls with Carbon Nanotubes via In Situ Polymerization of Dopamine
}

\author{
Lin Rong, ${ }^{1}$ Xiaoqing Mu, ${ }^{2}$ Jinchao Zhao $\mathbb{D}^{3},{ }^{3}$ Leping Huang $\left(\mathbb{D},{ }^{2}\right.$ Mingqiao Ye, ${ }^{1}$ and Na Wang \\ ${ }^{1}$ China Tobacco Hubei Industrial Co. Ltd, Wuhan 430040, China \\ ${ }^{2}$ School of Material Science and Engineering, Wuhan Textile University, Wuhan 430200, China \\ ${ }^{3}$ Hubei Key Laboratory of Biomass Fibers and Eco-dyeing \& Finishing, Wuhan Textile University, Wuhan 430200, China \\ Correspondence should be addressed to Jinchao Zhao; jczhao@wtu.edu.cn and Leping Huang; lphuang@wtu.edu.cn
}

Received 7 May 2021; Accepted 23 July 2021; Published 20 August 2021

Academic Editor: Marco Rossi

Copyright (C) 2021 Lin Rong et al. This is an open access article distributed under the Creative Commons Attribution License, which permits unrestricted use, distribution, and reproduction in any medium, provided the original work is properly cited.

Millimeter-scale calcium alginate aqueous core capsules (mm-CaSA-Caps) are suitable for embedding of temperature and chemical sensitive substances because of its excellent biocompatibility and biodegradability. In this study, mm-CaSA-Caps were coated with multiwalled carbon nanotubes (MWNTs) via in situ self-polymerization of dopamine (DA) under mild conditions. During the modification process, $\mathrm{mm}$-CaSA-Caps transferred quickly from colorless and transparent capsules to dark and opaque "pearls" in $15 \mathrm{~min}$. The obtained MWNTs-polydopamine- (PDA-) modified mm-CaSA-Caps (mm-MWNTs-PDA@CaSA-Caps) retained the spherical appearance of mm-CaSA-Caps with uniform coating of MWNTs-PDA. Obviously, the MWNTs were easily coated on the mm-PDA@CaSA-Caps due to the strong adhesive property of PDA. As the MWNTs content increased, the stacking density of MWNTs on surface of the mm-MWNTs-PDA@CaSA-Caps raised. The water loss ratio of mm-MWNTs-PDA@CaSACaps was enhanced ascribed to increasing the path length of water by raising stacking density of MWNTs. This study provided a new path for enhancement of the barrier property of hydrogel capsules.

\section{Introduction}

Compared with microscale and nanoscale capsules, millimeter-scale capsules own high encapsulation efficiency, uniform monodispersity, and excellent dimensional stability $[1,2]$ and have important application in high energy physics, biological medicine, construction and decoration, food, and cosmetics engineering. The conventional preparation method of microscale and nanoscale capsules, such as emulsion polymerization and duplicate-lattice method, cannot be used to fabricate the millimeter-scale capsules. The microfluidic method $[3,4]$, orifice-bath coagulation method $[5,6]$, liquid marbles method $[7,8]$, and layer by layer selfassembly method [9] have been developed.

Millimeter-scale calcium alginate aqueous core capsules (mm-CaSA-Caps) which have excellent biocompatibility and biodegradability are suitable for embedding of temperature and chemical sensitive substances [10]. The mmCaSA-Caps can be prepared with the orifice-bath coagulation method under mild reaction conditions $[6,11,12]$ that the droplets containing calcium ion are dripped vertically to coagulating bath of alginate solution at room temperature. Size and sphericity of mm-CaSA-Caps can be controlled by regulating parameters of solution (concentration, surface tension, viscosity, and density) and parameter of equipment (extrusion velocity, diameter of dripper, and drop height).

Calcium alginate capsules can swell slightly in acidic gastric solution, while they can be rapidly degraded in alkaline intestinal solution, which are used as intestinal agents for controlled release [10]. In addition, the degradation rate of calcium alginate capsules at $60^{\circ} \mathrm{C}$ is accelerated. Because of its temperature sensitivity, calcium alginate microspheres encapsulated with gold nanoparticles via the in situ method can degrade and release drugs slowly if the temperature of microbulb suspension raises above $60^{\circ} \mathrm{C}$ through infrared laser irradiation. It can be used in the photothermal therapy and controlling chemotherapy of tumors [13]. However, the surface modification methods of calcium alginate capsules are limited by its $\mathrm{pH}$ and temperature sensitivity [13]. Physical blending [2] and ion crosslinking [14] are usually 
used in surface modification of calcium alginate capsules at room temperature.

This study took advantage of the mild reactivity, strong adhesion, and good biocompatibility [15] of polydopamine (PDA). Multiwalled carbon nanotubes (MWNTs) were coated on the surface of mm-CaSA-Caps via in situ crosslinking with dopamine (DA) under conditions of room temperature and weak alkaline. The effect of MWNTs concentrations on the controlled release performance of $\mathrm{mm}$-CaSA-Caps was investigated.

\section{Materials and Methods}

2.1. Materials. Sodium alginate (SA, $10 \mathrm{~g} \cdot \mathrm{L}^{-1}, 20^{\circ} \mathrm{C}$, $\geq 20 \mathrm{MPa} \cdot \mathrm{s}$ ) and sodium carboxymethyl cellulose (CMC, $\left.20 \mathrm{~g} \cdot \mathrm{L}^{-1}, 25^{\circ} \mathrm{C}, 800-1200 \mathrm{MPa} \cdot \mathrm{s}\right)$ were chemically pure and purchased from Sinopharm Chemical Reagent Co., Ltd., China. Disodium hydrogen phosphate dodecahydrate $\left(\mathrm{Na}_{2} \mathrm{HPO}_{4} \cdot 12 \mathrm{H}_{2} \mathrm{O}\right)$, citric acid monohydrate $\left(\mathrm{C}_{6} \mathrm{H}_{8} \mathrm{O}_{7} \cdot \mathrm{H}_{2} \mathrm{O}\right)$, calcium chloride $\left(\mathrm{CaCl}_{2}\right)$, ammonium persulfate (AP), and sodium periodate (SP) were analytically pure and purchased from Sinopharm Chemical Reagent Co., Ltd., China. Hydrochloric acid was analytically pure and purchased from Xinyang Chemical Reagent Factory. Tris (hydroxymethyl) aminomethane (Trisbase) was obtained from Guangzhou Saiguo Biotech Co., Ltd., China. Dopamine was purchased from Macklin, Shanghai, China. Multiwalled carbon nanotubes (MWNTs) with diameters ranging from 10 to $20 \mathrm{~nm}$ and lengths ranging from 10 to $30 \mu \mathrm{m}$ were purchased from Beijing Deke Daojin Science and Technology Co., Ltd.

2.2. Preparation of mm-CaSA-Caps. Referring to the previous work of our research group, $2.01 \mathrm{~g}$ SA swelled in $400 \mathrm{~mL}$ deionized water at room temperature for $4 \mathrm{~h}$ and stirred at $55^{\circ} \mathrm{C}$ for $6 \mathrm{~h}$. Then, $0.5^{\circ} \mathrm{wt} \%$ SA solution was obtained. $6.09 \mathrm{~g} \mathrm{CMC}$ swelled in $400 \mathrm{~mL}$ deionized water at room temperature for $4 \mathrm{~h}$ and stirred at $90^{\circ} \mathrm{C}$ for $6 \mathrm{~h} .1 .5^{\circ} \mathrm{wt}$ $\%$ CMC solution was obtained after standing and defoaming for $24 \mathrm{~h}$. $4.04 \mathrm{~g} \mathrm{CaCl}_{2}$ was dissolved in $1.5^{\circ} \mathrm{wt} \%$ $\mathrm{CMC}$ solution with stirring. $1.5^{\circ} \mathrm{wt} \% \mathrm{CMC} / 1.0^{\circ} \mathrm{wt} \% \mathrm{CaCl}_{2}$ mixed solution was obtained after standing and defoaming for $24 \mathrm{~h}$.

$1.5^{\circ} \mathrm{wt} \% \mathrm{CMC} / 1.0^{\circ} \mathrm{wt} \% \mathrm{CaCl}_{2}$ solution was loaded into a plastic syringe $(20 \mathrm{~mL})$ and pumped dropwise vertically with a flow rate of $0.5 \mathrm{~mL} \cdot \mathrm{min}^{-1}$ by a dripping nozzle into continuously stirred $200 \mathrm{~mL} 0.5^{\circ} \mathrm{wt} \% \mathrm{SA}$ solution. Inner and outer diameter of the dripping nozzle was $0.41 / 0.71 \mathrm{~mm}$. The distance between the dripping nozzle and the surface of SA solution was $10 \mathrm{~cm}$ fixed. The stirring speed was $200^{\circ} \mathrm{r} \cdot \mathrm{min}^{-1}$ for $15 \mathrm{~min}$, and the reaction continued for $15 \mathrm{~min}$ after dripping. The obtained capsules were washed with deionized water three times through a sieve. Then, an additional crosslinking process for capsules was conducted for $15 \mathrm{~min}$ in $100 \mathrm{~mL} 2.0^{\circ} \mathrm{wt} \% \mathrm{CaCl}_{2}$ solution. The mm-CaSA-Caps were obtained after washing with deionized water for three times. The above experiments were carried out at room temperature.
2.3. Preparation of mm-CaSA-Caps Coated with MWNTs via In Situ of DA (mm-MWNTs-PDA@CaSA-Caps). $0.061 \mathrm{~g}$ Trisbase was dissolved in $50 \mathrm{~mL}$ deionized water under magnetic stirring. $0.02 \mathrm{~g}$ SDBS and a certain amount of MWNTs were added to the above solution under magnetic stirring for $30 \mathrm{~min}$. The solution was dispersed by an ultrasonic cell disruptor (JY96-IIN, Shanghai LNB Instrument Co., Ltd., China) with $1000 \mathrm{~W}$ power for 30 min. $0.056 \mathrm{~g}$ SP was added, and hydrochloric acid was added to adjust $\mathrm{pH}$ to $8.5 .250 \mathrm{~mm}$-CaSA-Caps and $0.1 \mathrm{~g}$ DA were added to the above solution and then were stirred at room temperature for $24 \mathrm{~h}$. Then, the $\mathrm{mm}$ MWNTs-PDA@CaSA-Caps were obtained after filtering. MWNTs concentrations were $0.1,0.4,0.6$, and $1.0 \mathrm{~g} \cdot \mathrm{L}^{-1}$, respectively.

The preparation process and structure of mm-MWNTsPDA@CaSA-Caps are shown in Figures 1(a) and 1(b), respectively.

2.4. Properties and Characterizations of Samples. The reaction degree of DA in the reaction system was monitored with UV-vis spectrophotometry (TU-1900, Beijing Pullout General Instrument Co., Ltd., China). The wavelength ranged from $250 \mathrm{~nm}$ to $700 \mathrm{~nm}$. During the reaction process, $1 \mathrm{~mL}$ solution in the reaction system was diluted to $25 \mathrm{~mL}$ with deionized water at certain times, respectively. The images of capsules were captured with a digital camera (BLSM500, Jinhua Oushilang Trade Co., Ltd., China). The water on the surface of capsules was dried with filter paper and freeze-dried for $24 \mathrm{~h}$. The surface morphology of capsules was observed with a scanning electron microscope (SEM, JSM-IT300, JEOL Ltd., Japan).

20 capsules were selected randomly. The diameter of capsules was measured with a digital display vernier caliper, and the average of diameter was calculated. The sphericity (SF) of the water bead can be calculated by the following equation $[16,17]$ :

$$
\mathrm{SF}=\frac{\left(D_{\max }-D_{\min }\right)}{\left(D_{\max }+D_{\min }\right)}
$$

where $D_{\max }$ is the maximum diameter, and $D_{\min }$ is the minimum diameter perpendicular to $D_{\max }$. $\mathrm{SF}<0.07$ indicated a good sphericity.

20 capsules were selected randomly. In formula (2), $m_{0}$ is the initial weight of capsules and $m_{n}$ is the weight of capsules at a certain time of testing. The water loss ratio of capsules is

$$
\text { Water loss ratio }=\frac{m_{n}}{m_{0}} \times 100 \% .
$$

\section{Results and Discussion}

3.1. Surface Morphology Characterization and Mechanism Analysis. As shown in Figure 2, during the process of DA polymerization, the colorless transparent mm-CaSA-Caps became black translucent mm-MWNTs-PDA@CaSA-Caps rapidly within $2 \mathrm{~min}$, and then, they became opaque within $15 \mathrm{~min}$. The black color gradually deepened as the reaction 


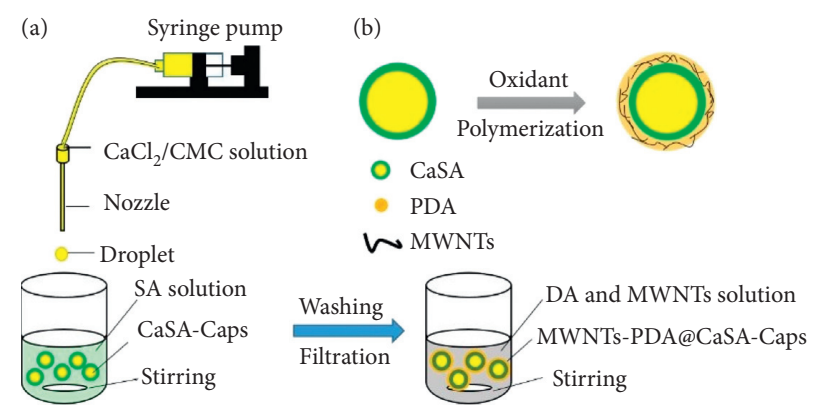

Figure 1: (a) Preparation of mm-MWNTs-PDA@CaSA-Caps. (b) Structure of mm-MWNTs-PDA@CaSA-Caps.

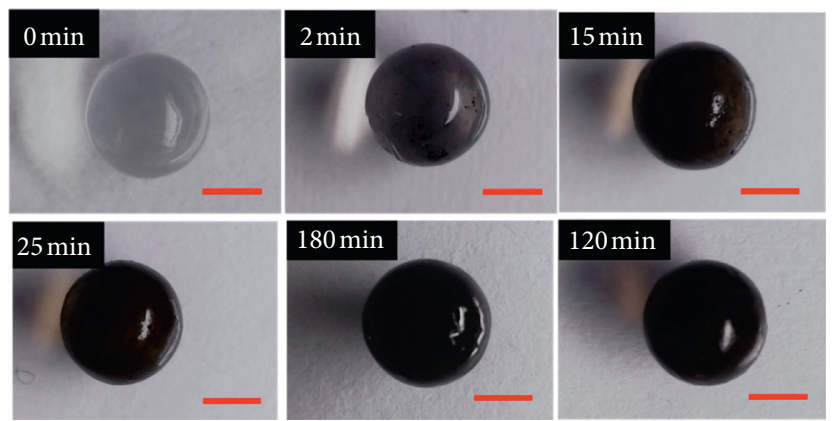

FIGURE 2: Images of mm-MWNTs-PDA@CaSA-Caps during the reaction process. Scale bar is $2 \mathrm{~mm}$.

continued. The UV absorption spectrum curves of the solution system during the reaction process (Figure 3(a)) showed that DA $(280 \mathrm{~nm})$ was oxidized to dopamine quinone $(350 \mathrm{~nm})$ first in an alkaline solution containing oxidants.

The rate of DA polymerization was expressed by the UV absorption peak of dopamine quinone $(350 \mathrm{~nm})$ with the change of its concentration [18]. Combined with the change of absorbance at $350 \mathrm{~nm}$ in the reaction process (Figure 3(b)), the concentration of dopamine quinone increased rapidly in the first 15 minutes and slowed down after 15 minutes. It showed that under room temperature and alkaline conditions, DA polymerized into PDA quickly [19, 20], which deposited on the surface of capsules. Then, MWNTs were attached to the surface of capsules due to the adhesion of PDA, and mm-MWNTs-PDA@CaSA-Caps were obtained.

3.2. Effect of MWNTs Concentration on Morphology and Properties of Capsules. The effect of MWNTs concentration on the morphology of mm-MWNTs-PDA@CaSA-Caps was further investigated. As given in Table 1, all of the colorless transparency mm-CaSA-Caps became black opaque within $300 \mathrm{~min}$ with various MWNTs concentrations. At the same reaction time, especially at the reaction time of $2 \mathrm{~min}$ and $15 \mathrm{~min}$, when the capsules were still black and translucent, obvious particles were aggregated on the surface of capsules with the increase of MWNTs concentrations. It indicated that MWNTs were successfully adhered to capsules due to the strong adhesion of PDA. In Figure 4, surface morphology of mmMWNTs-PDA@CaSA-Caps shows that MWNTs are attached on the surface of capsules. The distribution density of MWNTs on the surface of capsules increased with MWNTs concentrations increasing.

The effect of reaction time on diameter of mm-MWNTsPDA@CaSA-Caps is shown in Figure 5(a). The diameter of capsules increased gradually as the reaction time increased. The diameter grew faster within the first 15 minutes; it slowed down to a plateau after 15 minutes. In addition, with MWNTs concentrations increasing, capsules diameter grew faster. When the MWNTs concentration was $0.1 \mathrm{~g} \cdot \mathrm{L}^{-1}$, the diameter increased from $3.71 \pm 0.05 \mathrm{~mm}$ to $3.95 \pm 0.07 \mathrm{~mm}$ during reaction time of $0-540 \mathrm{~min}$. When the MWNTs concentration was $1.0 \mathrm{~g} \cdot \mathrm{L}^{-1}$, the diameter increased from $3.71 \pm 0.03 \mathrm{~mm}$ to $4.10 \pm 0.04 \mathrm{~mm}$ during the same reaction time. Under alkaline condition, the oxidant of SP accelerated the DA polymerization to PDA, and the generation speed of PDA was the fastest in the first $15 \mathrm{~min}$. At the same time, PDA-MWNTs composite layer formed on the surface of capsules, which was consistent with the report that PDA could be used as an interface binder to bond inorganic nanoparticles to any interface due to its strong adhesive property [21]. With the increase of MWNTs concentration, the density of MWNTs coated on the surface of capsules and the thickness of wall increased. Figure 5(b) shows the effect of MWNTs concentrations on SF of mm-MWNTs-PDA@ CaSA-Caps prepared. The SF value of capsules was 0.003-0.013 and far less than 0.07, indicating that PDA and MWNTs had no obvious effects on the sphericity of capsules. 


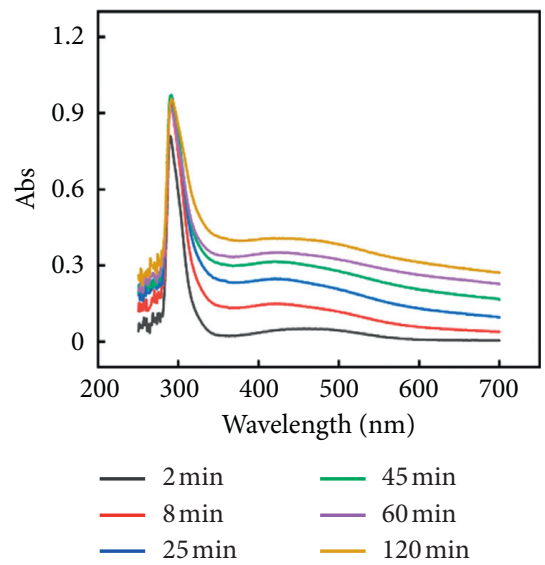

(a)

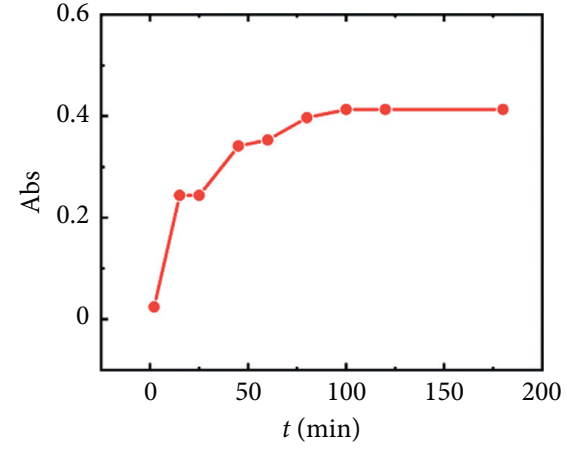

(b)

Figure 3: (a) The UV-vis spectra of DA solutions. (b) Absorption intensity in $350 \mathrm{~nm}$ during the reaction process.

TABLE 1: Effect of MWNTs concentration on morphology of capsules during the reaction. The scales bar in photos is $2 \mathrm{~mm}$.

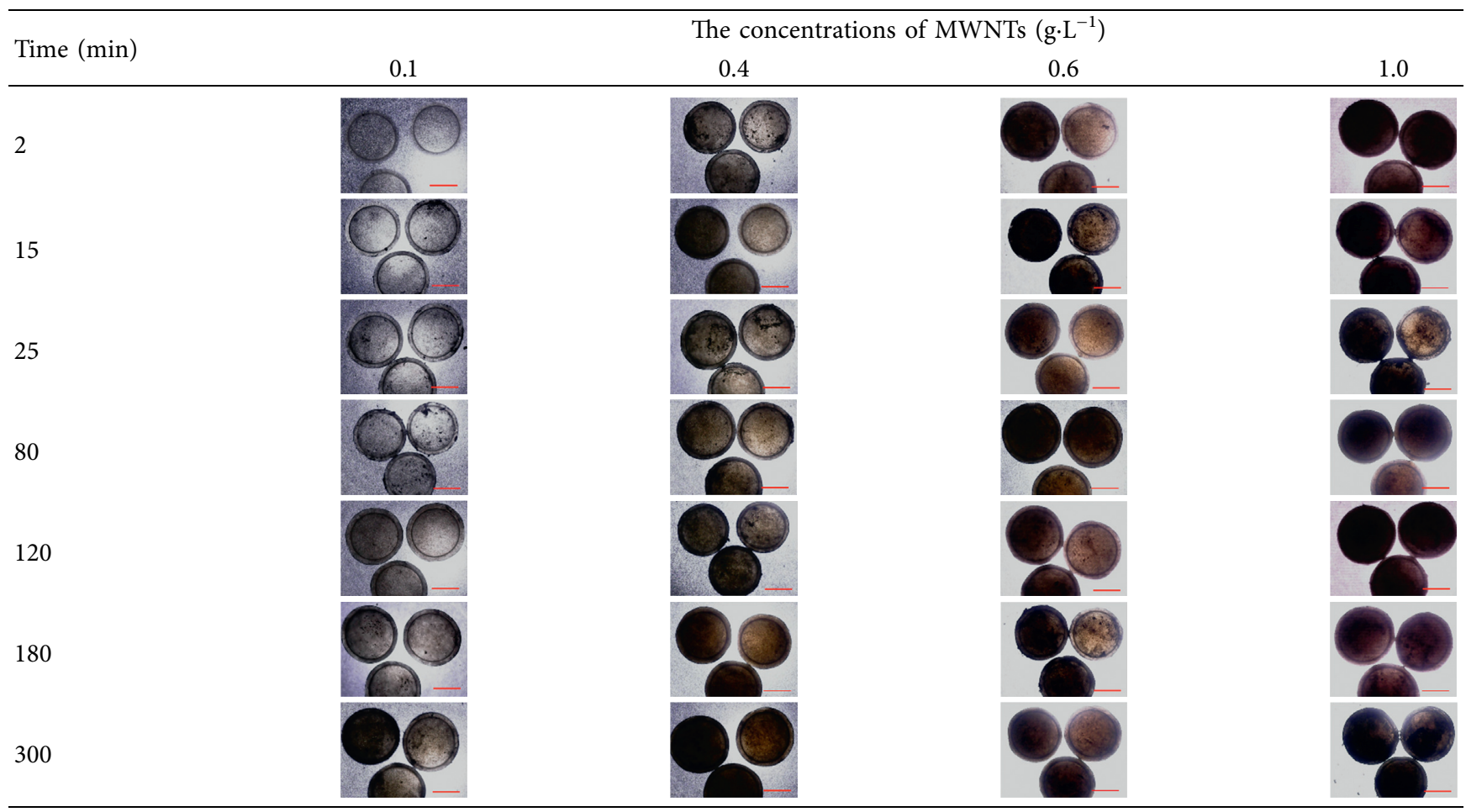

Figure 6 shows the effect of MWNTs concentrations on water loss ratio of mm-MWNTs-PDA@CaSA-Caps. In Figure 6(a), the data points are too dense to distinguish in initial time, so the data from 0 to $120 \mathrm{~min}$ are listed alone in Figure 6(b). Compared with mm-CaSA-Caps, water loss ratio of mm-MWNTs-PDA@CaSA-Caps decreased with different MWNTs concentrations in $120 \mathrm{~min}$, indicating that the PDA-MWNTs composite layer could delay the water loss in capsules effectively, that is, the water storage stability of the mm-CaSA-Caps was improved. It also showed that water loss ratio of the mm-MWNTs-PDA@ CaSA-Caps with $0.1 \mathrm{~g} \cdot \mathrm{L}^{-1} \mathrm{MWNTs}$ had little difference with that of the mm-CaSA-Caps. Because the surface of mmCaSA-Caps could not be completely covered as the concentration of MWNTs was low. When the concentration of MWNTs increased, the water loss ratio of capsules decreased significantly in the same time. However, the water loss ratio of the mm-MWNTs-PDA@CaSA-Caps with $0.6 \mathrm{~g} \cdot \mathrm{L}^{-1} \mathrm{MWNTs}$ had little difference with that of the mmMWNTs-PDA@CaSA-Caps with 0.6 g. $\mathrm{L}^{-1}$ MWNTs. The 

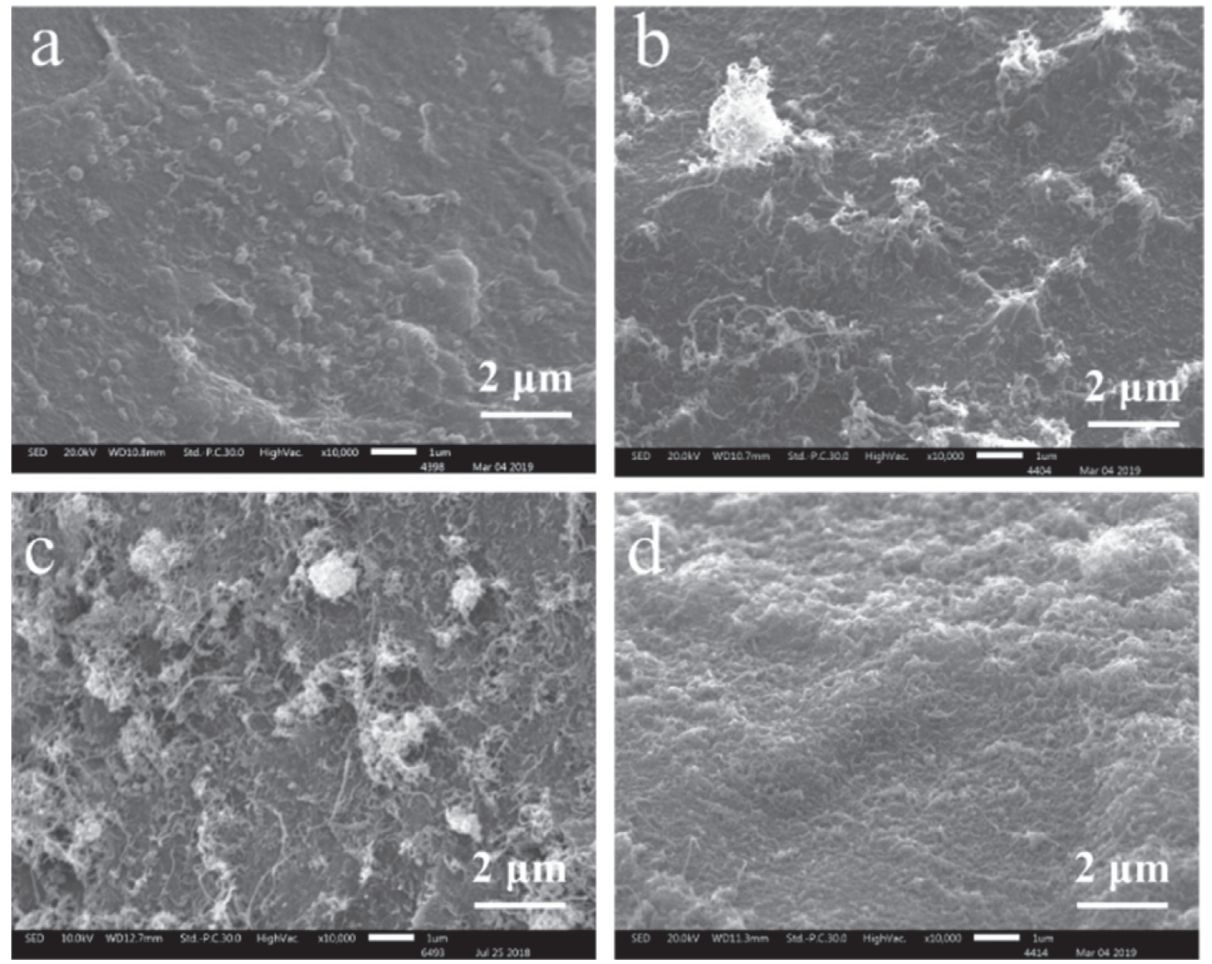

Figure 4: Surface morphology of mm-MWNTs-PDA@CaSA-Caps. MWNTs concentrations are (a) $0.1 \mathrm{~g} \cdot \mathrm{L}^{-1}$, (b) $0.4 \mathrm{~g} \cdot \mathrm{L}^{-1}$, (c) $0.6 \mathrm{~g} \cdot \mathrm{L}^{-1}$, and (d) $1.0 \mathrm{~g} \cdot \mathrm{L}^{-1}$.

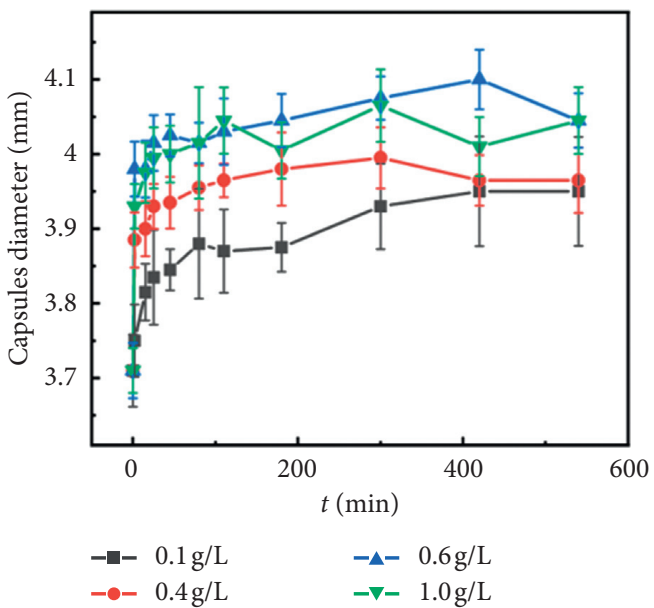

(a)

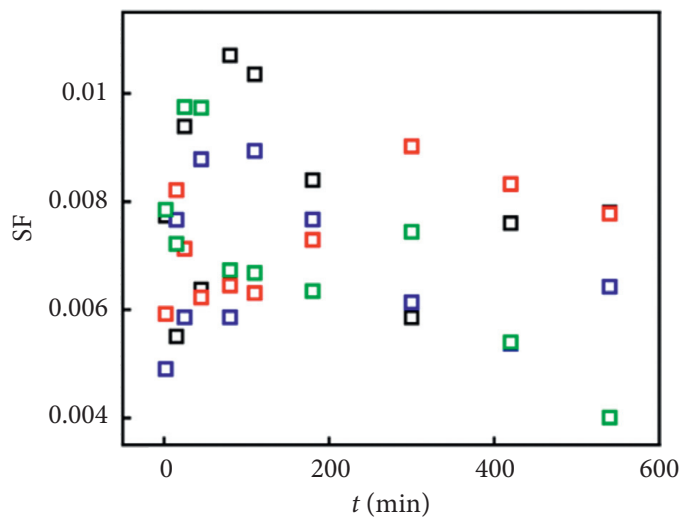

$\begin{array}{ll}0.1 \mathrm{~g} / \mathrm{L} & \square 0.6 \mathrm{~g} / \mathrm{L} \\ 0.4 \mathrm{~g} / \mathrm{L} & \square 1.0 \mathrm{~g} / \mathrm{L}\end{array}$

(b)

FIGURE 5: Effect of MWNTs concentration on (a) particle size and (b) SF of capsules during the reaction process.

reason was that the adsorption amount of MWNTs on mmCaSA-Caps reached saturation. It meant that the MWNTs concentration kept increasing; no more MWNTs could be adhered to the surface of capsules, which was also consistent with the results of capsule diameter. At test time of $540 \mathrm{~min}$, water loss ratios of capsules were $70.07 \%, 57.85 \%$, $52.29 \%, 45.88 \%$, and $47.11 \%$ with $0 \mathrm{~g} \cdot \mathrm{L}^{-1}, 0.1 \mathrm{~g} \cdot \mathrm{L}^{-1}$,
$0.4 \mathrm{~g} \cdot \mathrm{L}^{-1}, 0.6 \mathrm{~g} \cdot \mathrm{L}^{-1}$, and $1.0 \mathrm{~g} \cdot \mathrm{L}^{-1}$ MWNTs, respectively. The water loss ratios of the mm-MWNTs-PDA@CaSA-Caps decreased significantly. Since the MWNTs were attached on the surface on capsules by PDA which was prepared via the in situ polymerization method, the mm-MWNTs-PDA@ CaSA-Caps with a better water storage stability were obtained. 


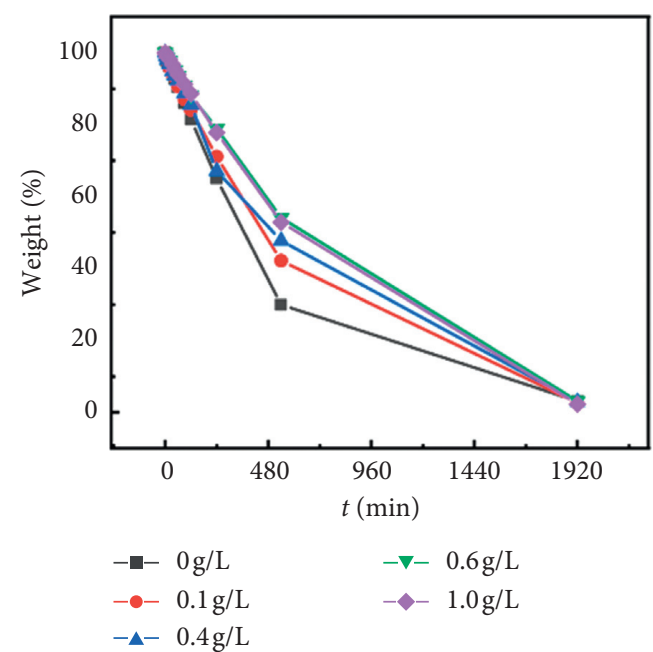

(a)

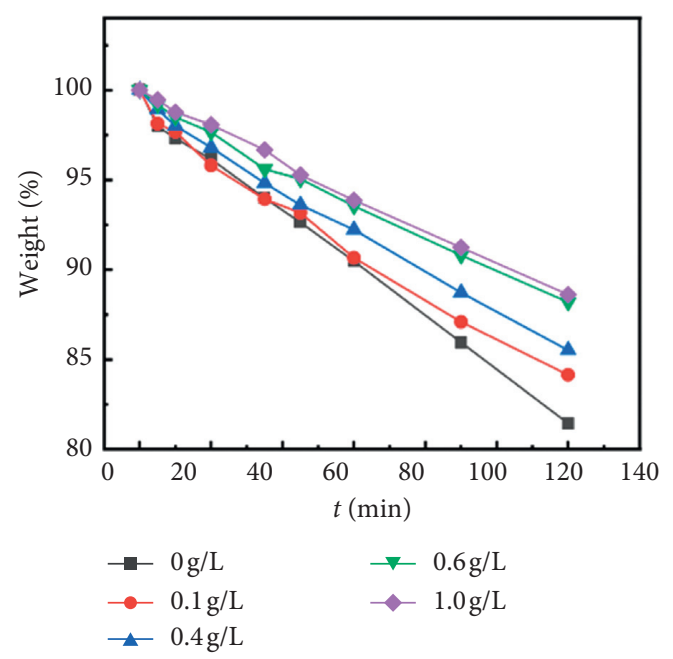

(b)

Figure 6: Effect of MWNTs concentration on water loss ratio of capsules at (a) test time 0-1920 min and (b) test time 0-120 min.

\section{Conclusions}

In this study, inorganic nanomaterials carbon nanotubes with special properties and strong adhesion property of PDA were used to modify the surface of $\mathrm{mm}$-CaSA-Caps via the in situ polymerization method at room temperature and under mild conditions. The mm-MWNTs-PDA@CaSA-Caps were obtained. The results showed PDA was successfully deposited on the surface of mm-CaSA-Caps, and MWNTs could be further attached to the surface of capsules. The MWNTs-PDA barrier layer was formed on the surface of mm-CaSA-Caps, which prevented core solution from loss effectively and prolonged the storage period of $\mathrm{mm}$-CaSACaps. When MWNTs concentration was $0.6 \mathrm{~g} \cdot \mathrm{L}^{-1}$, the water storage stability of mm-MWNTs-PDA@CaSA-Caps was the best.

\section{Data Availability}

The data used to support the findings of this study are available from the corresponding author upon request.

\section{Conflicts of Interest}

The authors declare that there are no conflicts of interest.

\section{Acknowledgments}

The authors would like to acknowledge the financial support from the National Natural Science Foundation of China (51303138).

\section{References}

[1] A. Blandino, M. Macías, and D. Cantero, "Glucose oxidase release from calcium alginate gel capsules," Enzyme and Microbial Technology, vol. 27, no. 3-5, pp. 319-324, 2000.

[2] J.-Y. Wang, Y. Jin, R. Xie et al., "Novel calcium-alginate capsules with aqueous core and thermo-responsive membrane," Journal of Colloid and Interface Science, vol. 353, no. 1, pp. 61-68, 2011.

[3] A. K. Tucker-Schwartz, Z. Bei, R. L. Garrell, and T. B. Jones, "Polymerization of electric field-centered double emulsion droplets to create polyacrylate shells," Langmuir, vol. 26, no. 24, pp. 18606-18611, 2010.

[4] T. Shao, X. Feng, Y. Jin, and Y. Cheng, "Controlled production of double emulsions in dual-coaxial capillaries device for millimeter-scale hollow polymer spheres," Chemical Engineering Science, vol. 104, pp. 55-63, 2013.

[5] J. Zhao, Q. Guo, W. Huang et al., "Shape tuning and size prediction of millimeter-scale calcium-alginate capsules with aqueous core," Polymers, vol. 12, no. 3, pp. 688-704, 2020.

[6] C. Wischnewski, E. Zwar, H. Rehage, and J. Kierfeld, "Strong deformation of ferrofluid-filled elastic alginate capsules in inhomogenous magnetic fields," Langmuir, vol. 34, no. 45, pp. 13534-13543, 2018.

[7] K. Ueno, S. Hamasaki, E. J. Wanless, Y. Nakamura, and S. Fujii, "Microcapsules fabricated from liquid marbles stabilized with latex particles," Langmuir, vol. 30, no. 11, pp. 3051-3059, 2014.

[8] T. Takei, Y. Yamasaki, Y. Yuji et al., "Millimeter-sized capsules prepared using liquid marbles: encapsulation of ingredients with high efficiency and preparation of spherical core-shell capsules with highly uniform shell thickness using centrifugal force," Journal of Colloid and Interface Science, vol. 536, pp. 414-423, 2019.

[9] F. Caruso, "Hollow inorganic capsules via colloid-templated layer-by-layer electrostatic assembly," Topics in Current Chemistry, vol. 227, pp. 145-168, 2003.

[10] P. Wang, M. Li, D. Wei et al., "Electrosprayed soft capsules of millimeter size for specifically delivering fish oil/nutrients to the stomach and intestines," ACS Applied Materials \& Interfaces, vol. 12, no. 5, pp. 6536-6545, 2020.

[11] L. Huang, K. Wu, R. Zhang, and H. Ji, "Fabrication of multicore milli- and microcapsules for controlling hydrophobic drugs release using a facile approach," Industrial \& Engineering Chemistry Research, vol. 58, no. 36, pp. 1701717026, 2019.

[12] C. Arya, H. Oh, and S. R. Raghavan, “"Killer” microcapsules that can selectively destroy target microparticles in their 
vicinity," ACS Applied Materials \& Interfaces, vol. 8, no. 43, pp. 29688-29695, 2016.

[13] Q. Zou, F. Hou, H. Wang, Y. Liao, Q. Wang, and Y. Yang, "Microfluidic one-step preparation of alginate microspheres encapsulated with in situ-formed bismuth sulfide nanoparticles and their photothermal effect," European Polymer Journal, vol. 115, pp. 282-289, 2019.

[14] C. Ribeiro, J. Borges, A. Costa, V. Gaspar, V. Bermudez, and J. Mano, "Preparation of well-dispersed chitosan/alginate hollow multilayered microcapsules for enhanced cellular internalization," Molecules, vol. 23, no. 3, pp. 625-641, 2018.

[15] Y. Liu, K. Ai, and L. Lu, "Polydopamine and its derivative materials: synthesis and promising applications in energy, environmental, and biomedical fields," Chemical Reviews, vol. 114, no. 9, pp. 5057-5115, 2014.

[16] J. W. Woo, H. J. Roh, H. D. Park et al., "Sphericity optimization of calcium alginate gel beads and the effects of processing conditions on their physical properties," Food Science and Biotechnology, vol. 16, no. 5, pp. 715-721, 2007.

[17] B.-B. Lee, E. S. Chan, E.-S. Chan, and P. Ravindra, "Calcium pectinate beads formation: shape and size analysis," Journal of Engineering and Technological Sciences, vol. 46, no. 1, pp. 78-92, 2014.

[18] Q. Wei, F. Zhang, J. Li, B. Li, and C. Zhao, "Oxidant-induced dopamine polymerization for multifunctional coatings," Polymer Chemistry, vol. 1, no. 9, pp. 1430-1433, 2010.

[19] H. Lee, S. M. Dellatore, W. M. Miller, and P. B. Messersmith, "Mussel-inspired surface chemistry for multifunctional coatings," Science, vol. 318, no. 5849, pp. 426-430, 2007.

[20] C. Zhang, Y. Ou, W.-X. Lei, L.-S. Wan, J. Ji, and Z.-K. Xu, "CuSO4/H2O2-Induced rapid deposition of polydopamine coatings with high uniformity and enhanced stability," Angewandte Chemie International Edition, vol. 55, no. 9, pp. 3054-3057, 2016.

[21] T. Ding, L. Wang, J. Zhang, Y. Xing, and K. Cai, "Interfacially active polydopamine for nanoparticle stabilized nanocapsules in a one-pot assembly strategy toward efficient drug delivery," Journal of Materials Chemistry B, vol. 6, no. 12, pp. 1754-1763, 2018. 